\title{
Cauchy-Kowalevski Extensions and Monogenic Plane Waves in Clifford Analysis
}

\author{
Nele De Schepper and Frank Sommen
}

\begin{abstract}
This paper deals with axially and biaxial monogenic functions that are derived using two fundamental methods of Clifford analysis, namely the Cauchy-Kowalevski extension and monogenic plane waves.
\end{abstract}

Mathematics Subject Classification (2010). 30G35.

Keywords. Clifford analysis, CK-extension, monogenic plane wave, axially monogenic function, biaxial monogenic function, Cauchy-Riemann equation.

\section{Introduction}

Clifford analysis (see a.o. $[1,3]$ ) is a theory that offers a natural generalization of complex analysis to higher dimensions. To $\mathbb{R}^{m}$, the Euclidean space in $m$ dimensions, we first associate the Clifford algebra $C \ell_{0, m}$, generated by the canonical basis $e_{i}, i=1, \ldots, m$. These generators satisfy the multiplication rules $e_{i} e_{j}+e_{j} e_{i}=-2 \delta_{i j}$.

The Clifford algebra $C \ell_{0, m}$ can be decomposed as $C \ell_{0, m}=\oplus_{k=0}^{m} C \ell_{0, m}^{k}$ with $C \ell_{0, m}^{k}$ the space of $k$-vectors defined by

$$
C \ell_{0, m}^{k}=\operatorname{span}\left\{e_{i_{1} \cdots i_{k}}=e_{i_{1}} \cdots e_{i_{k}}, \quad i_{1}<\cdots<i_{k}\right\} .
$$

More precisely, we have that the space of 1 -vectors is given by $C \ell_{0, m}^{1}=$ $\operatorname{span}\left\{e_{i}, i=1, \ldots, m\right\}$ and it is obvious that this space is isomorphic with $\mathbb{R}^{m}$. The space of so-called bivectors is given explicitly by

$$
C \ell_{0, m}^{2}=\operatorname{span}\left\{e_{i j}=e_{i} e_{j}, i<j\right\} .
$$

Moreover, the sum of a scalar and a bivector is a so-called parabivector. In what follows, $[a]_{k}$ denotes the projection of $a \in C \ell_{0, m}$ on $C \ell_{0, m}^{k}$.

We identify the point $\left(x_{1}, \ldots, x_{m}\right)$ in $\mathbb{R}^{m}$ with the vector variable $\underline{x}$ given by $\underline{x}=\sum_{j=1}^{m} x_{j} e_{j}$. The Clifford product of two vectors splits into a 
scalar part and a bivector part: $\underline{x} \underline{y}=\underline{x} \bullet \underline{y}+\underline{x} \wedge \underline{y}$ with

$$
\begin{aligned}
& \underline{x} \bullet \underline{y}=-\langle\underline{x}, \underline{y}\rangle=-\sum_{j=1}^{m} x_{j} y_{j}=\frac{1}{2}(\underline{x} \underline{y}+\underline{y} \underline{x}), \\
& \underline{x} \wedge \underline{y}=\sum_{j<k} e_{j} e_{k}\left(x_{j} y_{k}-x_{k} y_{j}\right)=\frac{1}{2}(\underline{x} \underline{y}-\underline{y} \underline{x}) .
\end{aligned}
$$

It is interesting to note that the square of a vector variable $\underline{x}$ is scalarvalued and equals the norm squared up to a minus sign: $\underline{x}^{2}=-\langle\underline{x}, \underline{x}\rangle=-|\underline{x}|^{2}$.

In a similar way we introduce a first order vector differential operator by $\partial_{\underline{x}}=\sum_{j=1}^{m} \partial_{x_{j}} e_{j}$. This operator is the so-called Dirac operator. Its square equals, up to a minus sign, the Laplace operator in $\mathbb{R}^{m}: \partial_{\underline{x}}^{2}=-\Delta_{m}$. In the sequel, we will also consider the so-called Cauchy-Riemann operator $\partial_{x_{0}}+\partial_{\underline{x}}$ for which

$$
\left(\partial_{x_{0}}+\partial_{\underline{x}}\right)\left(\partial_{x_{0}}-\partial_{\underline{x}}\right)=\Delta_{m+1} .
$$

A function $f\left(x_{1}, \ldots, x_{m}\right)$, respectively $f\left(x_{0}, x_{1}, \ldots, x_{m}\right)$, defined and continuously differentiable in an open domain of $\mathbb{R}^{m}$, respectively $\mathbb{R}^{m+1}$, and taking values in $C \ell_{0, m}$, is called monogenic in that region if $\partial_{\underline{x}} f=0$, respectively $\left(\partial_{x_{0}}+\partial_{\underline{x}}\right) f=0$.

A monogenic function is called axially monogenic if it is of the "axial form"

$$
f\left(x_{0}, \underline{x}\right)=A\left(x_{0},|\underline{x}|\right)+\frac{\underline{x}}{|\underline{x}|} B\left(x_{0},|\underline{x}|\right),
$$

where $A$ and $B$ are scalar functions. The monogenicity condition

$$
\left(\partial_{x_{0}}+\partial_{\underline{x}}\right) f=0
$$

then leads to the Vekua system for $A$ and $B$ :

$$
\partial_{x_{0}} A-\partial_{\rho} B=\frac{m-1}{\rho} B, \quad \partial_{x_{0}} B+\partial_{\rho} A=0, \quad \rho=|\underline{x}|,
$$

the solution of which leads to numerous special monogenic functions (cf. [14]).

In general, every monogenic function $f\left(x_{0}, \underline{x}\right)$ is determined by its restriction $f(0, \underline{x})$ to the hyperplane $x_{0}=0$ and, conversely, any given real analytic function $f(\underline{x})$ has a monogenic extension $f\left(x_{0}, \underline{x}\right)$ called CauchyKowalevski extension (abbreviated CK-extension). Axial monogenics are also determined by their restriction $A\left(x_{0}, 0\right)$ to the $x_{0}$-axis and, consequently, starting from a given function $A\left(x_{0}\right)$ of one variable, one may establish its axial CK-extension

$$
A\left(x_{0},|\underline{x}|\right)+\frac{\underline{x}}{|\underline{x}|} B\left(x_{0},|\underline{x}|\right) .
$$

If one composes this operation with the restriction to $x_{0}=0$ we arrive at a correspondence

$$
A\left(x_{0}\right) \mapsto A(0,|\underline{x}|)+\frac{\underline{x}}{|\underline{x}|} B(0,|\underline{x}|),
$$

transforming one dimensional functions into higher dimensional ones. 
In Section 2, we study this axial CK-extension and the resulting correspondence in detail for some cases, namely $A\left(x_{0}\right)=e^{x_{0}}, A\left(x_{0}\right)=e^{-x_{0}^{2} / 2}$ and $A\left(x_{0}\right)=\left(1-x_{0}^{2}\right)^{\alpha}, \alpha \in \mathbb{R}$.

In Section 3, we generalize our result to the case of biaxial monogenic functions. These functions originate from the more general splitting $\mathbb{R}^{m}=\mathbb{R}^{p} \oplus \mathbb{R}^{q}$ giving rise to two vector variables $\underline{x}=\sum_{j=1}^{p} x_{j} e_{j}$ and $\underline{y}=\sum_{j=1}^{q} y_{j} e_{j+p}$ which anti-commute, and the corresponding Dirac operators

$$
\partial_{\underline{x}}=\sum_{j=1}^{p} \partial_{x_{j}} e_{j}, \quad \partial_{\underline{y}}=\sum_{j=1}^{q} \partial_{y_{j}} e_{j+p} .
$$

The over-all Dirac operator is $\partial_{\underline{x}}+\partial_{\underline{y}}$ and biaxial monogenics are defined as solutions of

$$
\left(\partial_{\underline{x}}+\partial_{\underline{y}}\right) f(\underline{x}, \underline{y})=0
$$

of the special form

$$
A+\frac{\underline{x}}{|\underline{x}|} B+\frac{\underline{y}}{|\underline{y}|} C+\frac{\underline{x} \underline{y}}{|\underline{x}||\underline{y}|} D,
$$

whereby $A, B, C, D$ are scalar functions of the two radial variables $|\underline{x}|$ and $|\underline{y}|$. Biaxial monogenics split into biaxial monogenics of vector type $\frac{\underline{x}}{|\underline{x}|} B+\frac{\underline{y}}{|\underline{y}|} C$ and of parabivector type $A+\frac{\underline{x} \underline{y}}{|\underline{x}||\underline{y}|} D$. It may be shown that (see [3]) the pairs $(A, D),(B, C)$ will also satisfy Vekua-type systems $(r=|\underline{x}|, \rho=|\underline{y}|)$

$$
\begin{gathered}
\partial_{r} A+\left(\partial_{\rho}+\frac{q-1}{\rho}\right) D=0 \\
\left(\partial_{r}+\frac{p-1}{r}\right) D-\partial_{\rho} A=0
\end{gathered}
$$

resp.

$$
\begin{aligned}
\left(\partial_{r}+\frac{p-1}{r}\right) B+\left(\partial_{\rho}+\frac{q-1}{\rho}\right) C & =0 \\
\partial_{r} C-\partial_{\rho} B & =0 .
\end{aligned}
$$

Similar to axial monogenics, biaxial monogenics are determined by their restriction to the first axis $\mathbb{R}^{p}$ given by $\underline{y}=\underline{0}$ and the second axis $\mathbb{R}^{q}$ given by $\underline{x}=\underline{0}$. This leads to a correspondence between functions on $\mathbb{R}^{p}$ and functions on $\overline{\mathbb{R}^{q}}$ that will be investigated in a number of examples.

In Section 4, we will produce special axial and biaxial monogenics as linear superpositions of plane wave type monogenic functions.

In the axial case we start from monogenic plane waves of the simple form $(1-i \underline{t}) h\left(x_{0}+i\langle\underline{x}, \underline{t}\rangle\right)$, whereby $\underline{t} \in S^{m-1}$ is a variable unit vector and $h$ is a classical holomorphic function of the complex variable $x_{0}+i\langle\underline{x}, \underline{t}\rangle$. For special examples of holomorphic functions $h$ we then consider the integral

$$
\int_{S^{m-1}}(1-i \underline{t}) h\left(x_{0}+i\langle\underline{x}, \underline{t}\rangle\right) d \underline{t}
$$


which may be evaluated using Funk-Hecke's theorem and which gives rise to an axial monogenic function whose restriction to the $x_{0}$-axis is, up to a constant, simply $h\left(x_{0}\right)$.

In the biaxial case, we start from plane wave functions of either form

$$
(\underline{t}+i \underline{s}) h(i\langle\underline{x}, \underline{t}\rangle-\langle\underline{y}, \underline{s}\rangle) \quad \text { or } \quad(1+i \underline{t} \underline{s}) h(i\langle\underline{x}, \underline{t}\rangle-\langle\underline{y}, \underline{s}\rangle)
$$

with $\underline{t} \in S^{p-1}, \underline{s} \in S^{q-1}$ being variable orthogonal unit vectors. After integration of such functions over the bi-sphere $(\underline{t}, \underline{s}) \in S^{p-1} \times S^{q-1}$, we obtain biaxial monogenics of vector-type and of parabivector-type, respectively. We work out these integrals explicitly for the exponential function $\exp (i\langle\underline{x}, \underline{t}\rangle-\langle\underline{y}, \underline{s}\rangle)$, giving rise to integral expressions for the Clifford-Bessel function of biaxial type.

It is clear that this paper deals with simple, though fundamental, examples of special monogenic functions and that many more special functions may be computed using these methods.

For an introduction to Clifford analysis we refer to $[1,3,5,6]$. Other papers dealing with axial and biaxial monogenics include $[2,8,9,11-13]$.

\section{Cauchy-Kowalevski extensions: the axial framework}

\subsection{General problem}

Any axially symmetric function may be written in the form

$$
f\left(x_{0}, \underline{x}\right)=\sum_{s=0}^{\infty} c_{s} \underline{x}^{s} F_{s}\left(x_{0}\right) W\left(x_{0}\right)
$$

with $F_{0}\left(x_{0}\right)=1$, whereby the coefficients $c_{s}$ are determined by

$$
\partial_{\underline{x}}\left[c_{s} \underline{x}^{s}\right]=c_{s-1} \underline{x}^{s-1}, \quad c_{0}=1 .
$$

For axially monogenic functions $\left(\partial_{x_{0}}+\partial_{\underline{x}}\right)\left[f\left(x_{0}, \underline{x}\right)\right]=0$, the question is to determine

$$
f(0, \underline{x})=\sum_{s=0}^{\infty} c_{s} \underline{x}^{s} F_{s}(0) W(0) .
$$

In other words, the restriction to $\mathbb{R}$ of the function $f$, which is monogenic in the whole of $\mathbb{R}^{m+1}$, is given by $f\left(x_{0}, \underline{0}\right)=W\left(x_{0}\right)$. The aim is to determine its restriction to $\mathbb{R}^{m}$, i.e., $f(0, \underline{x})$.

By means of induction and using the fundamental formula

$$
\partial_{\underline{x}}\left[\underline{x}^{s}\right]= \begin{cases}-s \underline{x}^{s-1} & \text { for } s \text { even, } \\ -(s-1+m) \underline{x}^{s-1} & \text { for } s \text { odd, }\end{cases}
$$

one can prove that the coefficients $c_{s}$ satisfying (2.1) take the form

$$
c_{2 \ell}=\frac{\Gamma\left(\frac{m}{2}\right)}{\Gamma\left(\frac{m}{2}+\ell\right) 2^{2 \ell} \ell !} \quad \text { and } \quad c_{2 \ell+1}=-\frac{\Gamma\left(\frac{m}{2}\right)}{\Gamma\left(\frac{m}{2}+\ell+1\right) 2^{2 \ell+1} \ell !} .
$$




\subsection{Examples}

2.2.1. $W\left(x_{0}\right)=e^{x_{0}}$. This example was already considered in [14]. Given

$$
f\left(x_{0}, \underline{x}\right)=\sum_{s=0}^{\infty} c_{s} \underline{x}^{s} B_{s}\left(x_{0}\right) e^{x_{0}}
$$

with $B_{0}\left(x_{0}\right)=1, c_{s}$ satisfying $(2.1)$ and $f$ being monogenic in $\mathbb{R}^{m+1}$, we look for $f(0, \underline{x})$.

The first step is to determine $B_{s}\left(x_{0}\right)$. From the monogenicity of $f$, we obtain consecutively

$$
\begin{aligned}
\left(\partial_{x_{0}}+\partial_{\underline{x}}\right)\left[\sum_{s=0}^{\infty} c_{s} \underline{x}^{s} B_{s}\left(x_{0}\right) e^{x_{0}}\right]=0 \\
\Longleftrightarrow \sum_{s=0}^{\infty} B_{s}^{\prime}\left(x_{0}\right) e^{x_{0}} c_{s} \underline{x}^{s}+\sum_{s=0}^{\infty} B_{s}\left(x_{0}\right) e^{x_{0}} c_{s} \underline{x}^{s}+\sum_{s=1}^{\infty} B_{s}\left(x_{0}\right) e^{x_{0}} c_{s-1} \underline{x}^{s-1}=0 \\
\Longleftrightarrow \sum_{s=0}^{\infty} B_{s}^{\prime}\left(x_{0}\right) e^{x_{0}} c_{s} \underline{x}^{s}+\sum_{s=0}^{\infty} B_{s}\left(x_{0}\right) e^{x_{0}} c_{s} \underline{x}^{s}+\sum_{s=0}^{\infty} B_{s+1}\left(x_{0}\right) e^{x_{0}} c_{s} \underline{x}^{s}=0
\end{aligned}
$$

where in the last series we have made the substitution $s^{\prime}=s-1$.

Hence, functions $B_{s}\left(x_{0}\right)$ must satisfy

$$
B_{s+1}\left(x_{0}\right)=-B_{s}\left(x_{0}\right)-B_{s}^{\prime}\left(x_{0}\right), \quad B_{0}\left(x_{0}\right)=1,
$$

from which we obtain that $B_{2 \ell}\left(x_{0}\right)=1$ and $B_{2 \ell+1}\left(x_{0}\right)=-1$.

Consequently, using spherical co-ordinates $\underline{x}=|\underline{x}| \underline{\omega}, \underline{\omega} \in S^{m-1}$, we find

$$
\begin{aligned}
& \sum_{s=0}^{\infty} c_{s} \underline{x}^{s} B_{s}(0) \\
& \quad=\sum_{\ell=0}^{\infty} c_{2 \ell} \underline{x}^{2 \ell}-\sum_{\ell=0}^{\infty} c_{2 \ell+1} \underline{x}^{2 \ell+1} \\
& \quad=\Gamma\left(\frac{m}{2}\right) \sum_{\ell=0}^{\infty} \frac{(-1)^{\ell}|\underline{x}|^{2 \ell}}{\Gamma\left(\frac{m}{2}+\ell\right) 2^{2 \ell} \ell !}+\Gamma\left(\frac{m}{2}\right) \frac{|\underline{x}|}{2} \sum_{\ell=0}^{\infty} \frac{(-1)^{\ell}|\underline{x}|^{2 \ell}}{\Gamma\left(\frac{m}{2}+\ell+1\right) 2^{2 \ell} \ell !} \underline{\omega} .
\end{aligned}
$$

Finally, taking into account that

$$
2^{\nu} \widetilde{J}_{\nu}(\rho)=\sum_{\ell=0}^{\infty} \frac{(-1)^{\ell} \rho^{2 \ell}}{\ell ! \Gamma(\nu+\ell+1) 2^{2 \ell}}
$$

we obtain

$$
f(0, \underline{x})=\sum_{s=0}^{\infty} c_{s} \underline{x}^{s} B_{s}(0)=\Gamma\left(\frac{m}{2}\right) 2^{m / 2-1}\left(\widetilde{J}_{m / 2-1}(|\underline{x}|)+\widetilde{J}_{m / 2}(|\underline{x}|) \underline{x}\right),
$$

which corresponds to the result obtained in [14].

Remark 2.1. Note that the full axial CK-extension $f\left(x_{0}, \underline{x}\right)$ takes the form:

$$
f\left(x_{0}, \underline{x}\right)=\Gamma\left(\frac{m}{2}\right) 2^{m / 2-1}\left(\widetilde{J}_{m / 2-1}(|\underline{x}|)+\widetilde{J}_{m / 2}(|\underline{x}|) \underline{x}\right) e^{x_{0}} .
$$


2.2.2. $W\left(x_{0}\right)=e^{-x_{0}^{2} / 2}$. As a second example, we consider for $W\left(x_{0}\right)$ the hermite weight function. Hence, given

$$
f\left(x_{0}, \underline{x}\right)=\sum_{s=0}^{\infty} c_{s} \underline{x}^{s} H_{s}\left(x_{0}\right) e^{-x_{0}^{2} / 2}
$$

with $H_{0}\left(x_{0}\right)=1, c_{s}$ again satisfying $(2.1)$ and $f$ being monogenic in $\mathbb{R}^{m+1}$, the aim is to determine $f(0, \underline{x})$.

In a similar way as in the first example, the monogenicity of $f$ in the whole of $\mathbb{R}^{m+1}$ leads to the following recurrence relation for the functions $H_{s}\left(x_{0}\right)$ :

$$
H_{s+1}\left(x_{0}\right)=x_{0} H_{s}\left(x_{0}\right)-H_{s}^{\prime}\left(x_{0}\right), \quad H_{0}\left(x_{0}\right)=1 .
$$

From this recurrence relation we observe that $H_{s}\left(x_{0}\right)$ are the classical Hermite polynomials on the real line associated with the weight function $e^{-x_{0}^{2} / 2}$, which are defined by the Rodrigues formula

$$
H_{s}\left(x_{0}\right)=e^{x_{0}^{2} / 2}\left(-\frac{d}{d x_{0}}\right)^{s}\left[e^{-x_{0}^{2} / 2}\right] .
$$

These polynomials satisfy (see e.g. [10, p. 250]):

$$
H_{2 \ell}(0)=\frac{(-1)^{\ell}(2 \ell) !}{\ell ! 2^{\ell}} \quad \text { and } \quad H_{2 \ell+1}(0)=0,
$$

which leads to

$$
f(0, \underline{x})=\sum_{s=0}^{\infty} c_{s} \underline{x}^{s} H_{s}(0)=\sum_{\ell=0}^{\infty} c_{2 \ell} \underline{x}^{2 \ell} H_{2 \ell}(0)=\sum_{\ell=0}^{\infty} \frac{\Gamma\left(\frac{m}{2}\right)}{\Gamma\left(\frac{m}{2}+\ell\right)} \frac{(2 \ell) !}{(\ell !)^{2}}\left(\frac{|\underline{x}|^{2}}{8}\right)^{\ell} .
$$

Taking into account (see e.g., [10, p. 3])

$$
\frac{\Gamma\left(\ell+\frac{1}{2}\right)}{\Gamma\left(\frac{1}{2}\right)}=2^{-2 \ell} \frac{(2 \ell) !}{\ell !},
$$

we finally arrive at

$$
f(0, \underline{x})=\frac{\Gamma\left(\frac{m}{2}\right)}{\Gamma\left(\frac{1}{2}\right)} \sum_{\ell=0}^{\infty} \frac{\Gamma\left(\ell+\frac{1}{2}\right)}{\Gamma\left(\ell+\frac{m}{2}\right)} \frac{1}{\ell !}\left(\frac{|\underline{x}|^{2}}{2}\right)^{\ell}={ }_{1} F_{1}\left(\frac{1}{2} ; \frac{m}{2} ; \frac{|\underline{x}|^{2}}{2}\right)
$$

where

$$
{ }_{1} F_{1}(a ; c ; z)=\frac{\Gamma(c)}{\Gamma(a)} \sum_{\ell=0}^{\infty} \frac{\Gamma(a+\ell)}{\Gamma(c+\ell)} \frac{z^{\ell}}{\ell !}
$$

is Kummer's function.

Remark 2.2. When $m=1$, we have $f(0, x)=e^{x^{2} / 2}$.

Let us now calculate an expression for the full axial CK-extension $f\left(x_{0}, \underline{x}\right)$ which we will use in subsection 4.3.2. 
Splitting series (2.4) into even and odd parts, we obtain

$$
\begin{aligned}
& f\left(x_{0}, \underline{x}\right)=\Gamma\left(\frac{m}{2}\right)\left(\sum_{\ell=0}^{\infty} \frac{(-1)^{\ell}|\underline{x}|^{2 \ell} H_{2 \ell}\left(x_{0}\right)}{\Gamma\left(\frac{m}{2}+\ell\right) 2^{2 \ell} \ell !}\right) e^{-x_{0}^{2} / 2} \\
&-\Gamma\left(\frac{m}{2}\right)\left(\sum_{\ell=0}^{\infty} \frac{(-1)^{\ell}|\underline{x}|^{2 \ell+1} H_{2 \ell+1}\left(x_{0}\right)}{\Gamma\left(\frac{m}{2}+\ell+1\right) 2^{2 \ell+1} \ell !}\right) \underline{\omega} e^{-x_{0}^{2} / 2}
\end{aligned}
$$

with $\underline{\omega}=\frac{\underline{x}}{|\underline{x}|} \in S^{m-1}$.

We will now rewrite both series over $\ell$ as series in $x_{0}$ instead of $|\underline{x}|$. Hereto we use the explicit expression of the classical Hermite polynomials (see e.g., $[10$, p. 250$])$ :

$$
H_{n}\left(x_{0}\right)=n ! \sum_{i=0}^{\left\lfloor\frac{n}{2}\right\rfloor} \frac{(-1)^{i} x_{0}^{n-2 i}}{i ! 2^{i}(n-2 i) !} .
$$

Hence, for the first series we obtain:

$$
\sum_{\ell=0}^{\infty} \frac{(-1)^{\ell}|\underline{x}|^{2 \ell} H_{2 \ell}\left(x_{0}\right)}{\Gamma\left(\frac{m}{2}+\ell\right) 2^{2 \ell} \ell !}=\sum_{\ell=0}^{\infty} \frac{(-1)^{\ell}|\underline{x}|^{2 \ell}}{\Gamma\left(\frac{m}{2}+\ell\right) 2^{2 \ell} \ell !}(2 \ell) ! \sum_{i=0}^{\ell} \frac{(-1)^{i} x_{0}^{2 \ell-2 i}}{i ! 2^{i}(2 \ell-2 i) !} .
$$

Substituting $j=\ell-i$ and changing the order of summation, we find

$$
\begin{aligned}
\sum_{\ell=0}^{\infty} \frac{(-1)^{\ell}|\underline{x}|^{2 \ell} H_{2 \ell}\left(x_{0}\right)}{\Gamma\left(\frac{m}{2}+\ell\right) 2^{2 \ell} \ell !} & =\sum_{\ell=0}^{\infty} \frac{(-1)^{\ell}|\underline{x}|^{2 \ell}}{\Gamma\left(\frac{m}{2}+\ell\right) 2^{2 \ell} \ell !}(2 \ell) ! \sum_{j=0}^{\ell} \frac{(-1)^{\ell-j} x_{0}^{2 j}}{(\ell-j) ! 2^{\ell-j}(2 j) !} \\
& =\sum_{j=0}^{\infty} \frac{(-1)^{j}}{(2 j) !} 2^{j}\left(\sum_{\ell=j}^{\infty} \frac{(2 \ell) !}{\Gamma\left(\frac{m}{2}+\ell\right) 2^{3 \ell} \ell !(\ell-j) !}|\underline{x}|^{2 \ell}\right) x_{0}^{2 j} .
\end{aligned}
$$

Next, we interchange the indices $\ell$ and $j$, and rewrite the above expression as

$$
\begin{aligned}
& \sum_{\ell=0}^{\infty} \frac{(-1)^{\ell}|\underline{x}|^{2 \ell} H_{2 \ell}\left(x_{0}\right)}{\Gamma\left(\frac{m}{2}+\ell\right) 2^{2 \ell} \ell !}= \\
& \quad \sum_{\ell=0}^{\infty} \frac{(-1)^{\ell}}{2^{2 \ell}(2 \ell) !}|\underline{x}|^{2 \ell}\left(\sum_{j=\ell}^{\infty} \frac{(2 j) !|\underline{x}|^{2 j-2 \ell}}{\Gamma\left(\frac{m}{2}+j\right) 2^{3 j-3 \ell} j !(j-\ell) !}\right) x_{0}^{2 \ell} .
\end{aligned}
$$

The series over $j$ yields the Kummer function. Indeed, changing the summation index as $i=j-\ell$ and using relation (2.5), we arrive at

$$
\begin{aligned}
\sum_{j=\ell}^{\infty} \frac{(2 j) !|\underline{x}|^{2 j-2 \ell}}{\Gamma\left(\frac{m}{2}+j\right) 2^{3 j-3 \ell} j !(j-\ell) !} & =\sum_{i=0}^{\infty} \frac{(2 i+2 \ell) !}{(i+\ell) !} \frac{|\underline{x}|^{2 i}}{\Gamma\left(\frac{m}{2}+i+\ell\right) 2^{3 i} i !} \\
& =\frac{2^{2 \ell}}{\sqrt{\pi}} \sum_{i=0}^{\infty} \frac{\Gamma\left(i+\ell+\frac{1}{2}\right)}{\Gamma\left(\frac{m}{2}+i+\ell\right)} \frac{1}{i !}\left(\frac{|\underline{x}|^{2}}{2}\right)^{i} \\
& =\frac{2^{2 \ell}}{\sqrt{\pi}} \frac{\Gamma\left(\ell+\frac{1}{2}\right)}{\Gamma\left(\frac{m}{2}+\ell\right)}{ }_{1} F_{1}\left(\ell+\frac{1}{2} ; \frac{m}{2}+\ell ; \frac{|x|^{2}}{2}\right) .
\end{aligned}
$$


Substituting the above result into (2.6) and using once more (2.5), we finally arrive at

$$
\sum_{\ell=0}^{\infty} \frac{(-1)^{\ell}|\underline{x}|^{2 \ell} H_{2 \ell}\left(x_{0}\right)}{\Gamma\left(\frac{m}{2}+\ell\right) 2^{2 \ell} \ell !}=\sum_{\ell=0}^{\infty} \frac{(-1)^{\ell}|\underline{x}|^{2 \ell}}{2^{2 \ell} \ell ! \Gamma\left(\frac{m}{2}+\ell\right)}{ }_{1} F_{1}\left(\ell+\frac{1}{2} ; \frac{m}{2}+\ell ; \frac{|\underline{x}|^{2}}{2}\right) x_{0}^{2 \ell} .
$$

In a similar way we find

$$
\begin{aligned}
& \sum_{\ell=0}^{\infty} \frac{(-1)^{\ell}|\underline{x}|^{2 \ell+1} H_{2 \ell+1}\left(x_{0}\right)}{\Gamma\left(\frac{m}{2}+\ell+1\right) 2^{2 \ell+1} \ell !} \\
& \quad=\sum_{\ell=0}^{\infty} \frac{(-1)^{\ell}|\underline{x}|^{2 \ell+1}}{2^{2 \ell+1} \ell ! \Gamma\left(\frac{m}{2}+\ell+1\right)}{ }_{1} F_{1}\left(\ell+\frac{3}{2} ; \frac{m}{2}+\ell+1 ; \frac{|\underline{x}|^{2}}{2}\right) x_{0}^{2 \ell+1} .
\end{aligned}
$$

We thus conclude that

$$
\begin{aligned}
& f\left(x_{0}, \underline{x}\right)= \\
& \Gamma\left(\frac{m}{2}\right)\left(\sum_{\ell=0}^{\infty} \frac{(-1)^{\ell}|\underline{x}|^{2 \ell}}{2^{2 \ell} \ell ! \Gamma\left(\frac{m}{2}+\ell\right)}{ }_{1} F_{1}\left(\ell+\frac{1}{2} ; \frac{m}{2}+\ell ; \frac{|\underline{x}|^{2}}{2}\right) x_{0}^{2 \ell}\right) e^{-x_{0}^{2} / 2} \\
&-\Gamma\left(\frac{m}{2}\right)\left(\sum_{\ell=0}^{\infty} \frac{(-1)^{\ell}|\underline{x}|^{2 \ell+1}}{2^{2 \ell+1} \ell ! \Gamma\left(\frac{m}{2}+\ell+1\right)}\right. \\
&\left.\quad \times{ }_{1} F_{1}\left(\ell+\frac{3}{2} ; \frac{m}{2}+\ell+1 ; \frac{|\underline{x}|^{2}}{2}\right) x_{0}^{2 \ell+1}\right) \underline{\omega} e^{-x_{0}^{2} / 2}
\end{aligned}
$$

2.2.3. $W\left(x_{0}\right)=\left(1-x_{0}^{2}\right)^{\alpha}, \alpha \in \mathbb{R}$. Next, we take for $W\left(x_{0}\right)$ the Gegenbauer weight function. The axial CK-extension $f\left(x_{0}, \underline{x}\right)$ now takes a slightly different form, namely

$$
f\left(x_{0}, \underline{x}\right)=\sum_{s=0}^{\infty} c_{s} \underline{x}^{s} G_{s}^{\alpha}\left(x_{0}\right)\left(1-x_{0}^{2}\right)^{\alpha-s} .
$$

Again we have that $G_{0}^{\alpha}\left(x_{0}\right)=1$ and that the coefficients $c_{s}$ satisfy (2.1). Hence,

$$
f\left(x_{0}, \underline{0}\right)=\left(1-x_{0}^{2}\right)^{\alpha} .
$$

The question is to first determine the functions $G_{s}^{\alpha}\left(x_{0}\right)$ and then to find the $\operatorname{sum} \sum_{s=0}^{\infty} c_{s} \underline{x}^{s} G_{s}^{\alpha}(0)$.

From the monogenicity relation

$$
\left(\partial_{x_{0}}+\partial_{\underline{x}}\right)\left[f\left(x_{0}, \underline{x}\right)\right]=0
$$

we derive the recurrence relation

$$
G_{s+1}^{\alpha}\left(x_{0}\right)=2(\alpha-s) x_{0} G_{s}^{\alpha}\left(x_{0}\right)-\left(1-x_{0}^{2}\right)\left(G_{s}^{\alpha}\right)^{\prime}\left(x_{0}\right), \quad G_{0}^{\alpha}\left(x_{0}\right)=1 .
$$


The lower degree polynomials take the form

$$
\begin{aligned}
& G_{0}^{\alpha}\left(x_{0}\right)=1, \\
& G_{1}^{\alpha}\left(x_{0}\right)=2 \alpha x_{0}, \\
& G_{2}^{\alpha}\left(x_{0}\right)=2 \alpha(2 \alpha-1) x_{0}^{2}-2 \alpha, \\
& G_{3}^{\alpha}\left(x_{0}\right)=4 \alpha(\alpha-1)(2 \alpha-1) x_{0}^{3}-12 \alpha(\alpha-1) x_{0},
\end{aligned}
$$

etc.

Putting

$$
G_{2 t}^{\alpha}\left(x_{0}\right)=\sum_{i=0}^{t} b_{2 i}^{2 t, \alpha} x_{0}^{2 i} \quad \text { and } \quad G_{2 t+1}^{\alpha}\left(x_{0}\right)=\sum_{i=0}^{t} b_{2 i+1}^{2 t+1, \alpha} x_{0}^{2 i+1},
$$

we then have the following result.

Theorem 2.3. For $0 \leq i \leq t$, one has

$$
\begin{aligned}
& b_{2 i}^{2 t, \alpha}=(-1)^{t} 2^{2 t}(2 t) ! \frac{(\alpha-2 t+1)_{2 t}}{(2 \alpha-4 t+1)_{2 t} \Gamma\left(\alpha-2 t+\frac{1}{2}\right)}(-1)^{i} \\
& \times \frac{2^{2 i} \Gamma\left(\alpha+\frac{1}{2}-t+i\right)}{(t-i) !(2 i) !} \\
& b_{2 i+1}^{2 t+1, \alpha}=(-1)^{t} 2^{2 t+1}(2 t+1) ! \frac{(\alpha-2 t)_{2 t+1}}{(2 \alpha-4 t-1)_{2 t+1} \Gamma\left(\alpha-2 t-\frac{1}{2}\right)}(-1)^{i} \\
& \times \frac{2^{2 i+1} \Gamma\left(\alpha+\frac{1}{2}-t+i\right)}{(t-i) !(2 i+1) !}
\end{aligned}
$$

with $(\alpha)_{\ell}=\alpha(\alpha+1) \cdots(\alpha+\ell-1)=\frac{\Gamma(\alpha+\ell)}{\Gamma(\alpha)}$, the Pochhammer's symbol.

Proof. This result is proved by induction on the degree of the polynomial and using the recurrence relation (2.8).

Using the above theorem, we can write $G_{t}^{\alpha}\left(x_{0}\right)$ in terms of the classical Gegenbauer polynomials on the real line.

Corollary 2.4. One has

$$
\begin{aligned}
G_{2 t}^{\alpha}\left(x_{0}\right) & =2^{2 t}(2 t) ! \frac{(\alpha-2 t+1)_{2 t}}{(2 \alpha-4 t+1)_{2 t}} C_{2 t}^{\alpha-2 t+\frac{1}{2}}\left(x_{0}\right), \\
G_{2 t+1}^{\alpha}\left(x_{0}\right) & =2^{2 t+1}(2 t+1) ! \frac{(\alpha-2 t)_{2 t+1}}{(2 \alpha-4 t-1)_{2 t+1}} C_{2 t+1}^{\alpha-2 t-\frac{1}{2}}\left(x_{0}\right),
\end{aligned}
$$

with $C_{n}^{\lambda}\left(x_{0}\right)$ being the classical Gegenbauer polynomial on the real line given explicitly by (see e.g., [10, p. 219])

$$
C_{n}^{\lambda}\left(x_{0}\right)=\frac{1}{\Gamma(\lambda)} \sum_{i=0}^{\left\lfloor\frac{n}{2}\right\rfloor} \frac{(-1)^{i} \Gamma(\lambda+n-i)}{i !(n-2 i) !}\left(2 x_{0}\right)^{n-2 i}, \quad \lambda>0 .
$$


Proof. This follows immediately from Theorem 2.3 and the explicit expression (2.9).

Now, we also easily obtain that $G_{2 t+1}^{\alpha}(0)=0$ and

$$
G_{2 t}^{\alpha}(0)=b_{0}^{2 t, \alpha}=(-1)^{t} 2^{2 t} \frac{(2 t) !}{t !} \frac{\Gamma(\alpha+1) \Gamma(2 \alpha-4 t+1) \Gamma\left(\alpha+\frac{1}{2}-t\right)}{\Gamma(\alpha-2 t+1) \Gamma(2 \alpha-2 t+1) \Gamma\left(\alpha-2 t+\frac{1}{2}\right)} .
$$

Taking into account (see e.g., [10, p. 3]):

$$
\Gamma(2 z)=\pi^{-\frac{1}{2}} 2^{2 z-1} \Gamma(z) \Gamma\left(z+\frac{1}{2}\right),
$$

the expression for $G_{2 t}^{\alpha}(0)$ can be simplified to

$$
G_{2 t}^{\alpha}(0)=(-1)^{t} \frac{(2 t) !}{t !} \frac{\Gamma(\alpha+1)}{\Gamma(\alpha-t+1)} .
$$

Hence, we find

$$
\begin{aligned}
\sum_{s=0}^{\infty} c_{s} \underline{x}^{s} G_{s}^{\alpha}(0) & =\sum_{\ell=0}^{\infty} c_{2 \ell} \underline{x}^{2 \ell} G_{2 \ell}^{\alpha}(0) \\
& =\sum_{\ell=0}^{\infty} \frac{\Gamma\left(\frac{m}{2}\right)}{\Gamma\left(\frac{m}{2}+\ell\right) 2^{2 \ell} \ell !}|\underline{x}|^{2 \ell} \frac{(2 \ell) !}{\ell !} \frac{\Gamma(\alpha+1)}{\Gamma(\alpha-\ell+1)}
\end{aligned}
$$

Using formula (2.5) and (see e.g., [10, p. 2])

$$
\Gamma(\alpha+1-\ell)=(-1)^{\ell} \Gamma(\alpha+1) \frac{\Gamma(-\alpha)}{\Gamma(\ell-\alpha)}
$$

expression (2.11) can be simplified to

$$
\begin{aligned}
\sum_{s=0}^{\infty} c_{s} \underline{x}^{s} G_{s}^{\alpha}(0) & =\sum_{\ell=0}^{\infty} \frac{\Gamma\left(\frac{m}{2}\right)}{\Gamma\left(\frac{m}{2}+\ell\right)} \frac{\Gamma\left(\ell+\frac{1}{2}\right)}{\Gamma\left(\frac{1}{2}\right)} \frac{\Gamma(\ell-\alpha)}{\Gamma(-\alpha)} \frac{\left(-|\underline{x}|^{2}\right)^{\ell}}{\ell !} \\
& ={ }_{2} F_{1}\left(\frac{1}{2},-\alpha ; m / 2 ;-|\underline{x}|^{2}\right)
\end{aligned}
$$

with

$$
{ }_{2} F_{1}(a, b ; c ; z)=\sum_{\ell=0}^{\infty} \frac{\Gamma(a+\ell)}{\Gamma(a)} \frac{\Gamma(b+\ell)}{\Gamma(b)} \frac{\Gamma(c)}{\Gamma(c+\ell)} \frac{z^{\ell}}{\ell !}
$$

being the hypergeometric function.

\section{Cauchy-Kowalevski-extensions: the biaxial framework}

In this section we consider functions of two vector variables $\underline{x}=\sum_{j=1}^{p} x_{j} e_{j}$ and $\underline{y}=\sum_{j=1}^{q} y_{j} e_{p+j}$. The sum $\underline{x}+\underline{y}$ represents a vector variable in $\mathbb{R}^{p+q}$ equipped with the orthonormal basis $\left(e_{1}, \ldots, e_{p}, e_{p+1}, \ldots, e_{p+q}\right)$. The corresponding Dirac operators take the form

$$
\partial_{\underline{x}}=\sum_{j=1}^{p} \partial_{x_{j}} e_{j} \quad \text { and } \quad \partial_{\underline{y}}=\sum_{j=1}^{q} \partial_{y_{j}} e_{p+j} .
$$


Note that the vector variables and Dirac operators anti-commute, i.e. $\underline{x} y=$ $-\underline{y} \underline{x}$ and $\partial_{\underline{x}} \partial_{\underline{y}}=-\partial_{\underline{y}} \partial_{\underline{x}}$ and that the vector variables are orthogonal, $\bar{i}$.e., $\langle\underline{x}, \underline{y}\rangle=0$.

In this biaxial framework we consider the analogue CK-problem as in the previous section.

\subsection{First example: Gauss function}

Given

$$
f(\underline{x}, \underline{y})=\sum_{s=0}^{\infty} c_{s} \underline{y}^{s} H_{s}(\underline{x}) e^{-|\underline{x}|^{2} / 2}
$$

with $H_{0}(\underline{x})=1, \partial_{\underline{y}}\left[c_{s} \underline{y}^{s}\right]=c_{s-1} \underline{y}^{s-1}, c_{0}=1$ and

$$
\left(\partial_{\underline{x}}+\partial_{\underline{y}}\right)[f(\underline{x}, \underline{y})]=0,
$$

the question is to determine

$$
f(\underline{0}, \underline{y})=\sum_{s=0}^{\infty} c_{s} \underline{y}^{s} H_{s}(\underline{0}) .
$$

In other words, the restriction to $\mathbb{R}^{p}$ of the function $f$, which is monogenic in the whole of $\mathbb{R}^{p+q}$, is given by the Gauss function: $f(\underline{x}, \underline{0})=e^{-|\underline{x}|^{2} / 2}$.

The aim is to determine its restriction to $\mathbb{R}^{q}$, i.e., to determine $f(\underline{0}, \underline{y})$.

In a completely similar way as in the previous section, we obtain that the coefficients $c_{s}$ are given by

$$
c_{2 \ell}=\frac{\Gamma\left(\frac{q}{2}\right)}{\Gamma\left(\frac{q}{2}+\ell\right) 2^{2 \ell} \ell !} \quad \text { and } \quad c_{2 \ell+1}=-\frac{\Gamma\left(\frac{q}{2}\right)}{\Gamma\left(\frac{q}{2}+\ell+1\right) 2^{2 \ell+1} \ell !} .
$$

From the monogenicity relation (3.1), we obtain consecutively

$$
\begin{gathered}
\left(\partial_{\underline{x}}+\partial_{\underline{y}}\right)\left[\sum_{s=0}^{\infty} c_{s} \underline{y}^{s} H_{s}(\underline{x}) e^{-|\underline{x}|^{2} / 2}\right]=0 \\
\Longleftrightarrow \sum_{s=0}^{\infty} c_{s}(-1)^{s} \underline{y}^{s}\left(-\underline{x} e^{-|\underline{x}|^{2} / 2} H_{s}(\underline{x})+e^{-|\underline{x}|^{2} / 2} \partial_{\underline{x}}\left[H_{s}(\underline{x})\right]\right) \\
+\sum_{s=1}^{\infty} c_{s-1} \underline{y}^{s-1} H_{s}(\underline{x}) e^{-|\underline{x}|^{2} / 2}=0 \\
\Longleftrightarrow \sum_{s=0}^{\infty} c_{s}(-1)^{s} \underline{y}^{s}\left(-\underline{x} H_{s}(\underline{x})+\partial_{\underline{x}}\left[H_{s}(\underline{x})\right]\right) e^{-|\underline{x}|^{2} / 2} \\
+\sum_{s=0}^{\infty} c_{s} \underline{y}^{s} H_{s+1}(\underline{x}) e^{-|\underline{x}|^{2} / 2}=0,
\end{gathered}
$$

hence the functions $H_{s}(\underline{x})$ satisfy the recurrence relation

$$
H_{s+1}(\underline{x})=(-1)^{s}\left(\underline{x}-\partial_{\underline{x}}\right)\left[H_{s}(\underline{x})\right], \quad H_{0}(\underline{x})=1 .
$$

From this recurrence relation we observe that

$$
H_{2 \ell}(\underline{x})=(-1)^{\ell} H_{2 \ell}^{R C H}(\underline{x}) \quad \text { and } \quad H_{2 \ell+1}(\underline{x})=(-1)^{\ell} H_{2 \ell+1}^{R C H}(\underline{x})
$$


with $H_{s}^{R C H}(\underline{x})$ being the so-called radial Clifford-Hermite polynomials which were introduced in [14]. Taking into account the expression of these polynomials in terms of the generalized Laguerre polynomials on the real line

$$
H_{2 \ell}^{R C H}(\underline{x})=2^{\ell} \ell ! L_{\ell}^{p / 2-1}\left(\frac{|\underline{x}|^{2}}{2}\right) ; \quad H_{2 \ell+1}^{R C H}(\underline{x})=2^{\ell} \ell ! L_{\ell}^{p / 2}\left(\frac{|\underline{x}|^{2}}{2}\right) \underline{x},
$$

and the relation (see e.g., [10, p. 240])

we find that

$$
L_{n}^{\alpha}(0)=\frac{(\alpha+1)_{n}}{n !}
$$

$$
H_{2 \ell}(\underline{0})=(-1)^{\ell} 2^{\ell} \frac{\Gamma\left(\frac{p}{2}+\ell\right)}{\Gamma\left(\frac{p}{2}\right)} \text { and } H_{2 \ell+1}(\underline{0})=0 .
$$

This finally yields

$$
\begin{aligned}
f(\underline{0}, \underline{y}) & =\sum_{s=0}^{\infty} c_{s} \underline{y}^{s} H_{s}(\underline{0})=\sum_{\ell=0}^{\infty} c_{2 \ell}\left(-|\underline{y}|^{2}\right)^{\ell} H_{2 \ell}(\underline{0}) \\
& =\frac{\Gamma\left(\frac{q}{2}\right)}{\Gamma\left(\frac{p}{2}\right)} \sum_{\ell=0}^{\infty} \frac{\Gamma\left(\frac{p}{2}+\ell\right)}{\Gamma\left(\frac{q}{2}+\ell\right)} \frac{1}{\ell !}\left(\frac{|\underline{y}|^{2}}{2}\right)^{\ell}={ }_{1} F_{1}\left(p / 2 ; q / 2 ;|\underline{y}|^{2} / 2\right) .
\end{aligned}
$$

Remark 3.1. Let us consider two special cases.

1. $\underline{p=q}$

In this case we again obtain a Gauss function, i.e., $f(\underline{0}, \underline{y})=e^{|\underline{y}|^{2} / 2}$, and as $f(\underline{x}, \underline{0})=e^{-|\underline{x}|^{2} / 2}$ we thus have a closed class.

2. $\underline{p-q=2 n, n \in \mathbb{N}}$

In this case we find

$$
\begin{aligned}
\frac{\Gamma\left(\frac{p}{2}+\ell\right)}{\Gamma\left(\frac{q}{2}+\ell\right)} & =\frac{\Gamma\left(\frac{q}{2}+\ell+n\right)}{\Gamma\left(\frac{q}{2}+\ell\right)}=\left(\frac{q}{2}+\ell\right)\left(\frac{q}{2}+\ell+1\right) \cdots\left(\frac{q}{2}+\ell+n-1\right) \\
& =P(\ell),
\end{aligned}
$$

$P(\ell)$ being a polynomial of degree $n$ in $\ell$. Consequently, we find that in this case

$$
f(\underline{0}, \underline{y})=\frac{\Gamma\left(\frac{q}{2}\right)}{\Gamma\left(\frac{p}{2}\right)} \sum_{\ell=0}^{\infty} P(\ell) \frac{1}{\ell !}\left(\frac{|\underline{y}|^{2}}{2}\right)^{\ell}=\frac{\Gamma\left(\frac{q}{2}\right)}{\Gamma\left(\frac{p}{2}\right)} P\left(\frac{E_{\underline{y}}}{2}\right)\left[e^{|\underline{y}|^{2} / 2}\right]
$$

with $E_{\underline{y}}=\left\langle\underline{y}, \partial_{\underline{y}}\right\rangle$ the Euler operator.

\subsection{Second example: Clifford-Bessel function of biaxial type}

In this subsection we consider the biaxial CK-extension of the Clifford-Bessel function without exponential factor, i.e., $\widetilde{J}_{p / 2-1}(|\underline{x}|)+\underline{x} \widetilde{J}_{p / 2}(|\underline{x}|)$. Put

$$
f(\underline{x}, \underline{y})=\sum_{s=0}^{\infty} c_{s} \underline{y}^{s} \partial_{\underline{x}}^{s}\left(\widetilde{J}_{p / 2-1}(|\underline{x}|)+\underline{x} \widetilde{J}_{p / 2}(|\underline{x}|)\right),
$$


where we take the coefficients $c_{s}$ such that $\sum_{s=0}^{\infty} c_{s} \underline{y}^{s} \partial_{\underline{x}}^{s}$ is monogenic, i.e.,

$$
\left(\partial_{\underline{x}}+\partial_{\underline{y}}\right)\left[\sum_{s=0}^{\infty} c_{s} \underline{y}^{s} \partial_{\underline{x}}^{s}\right]=0
$$

This leads to the equation

$$
c_{s} \partial_{\underline{y}}\left[\underline{y}^{s}\right]=(-1)^{s} c_{s-1} \underline{y}^{s-1} .
$$

Since

$$
f(\underline{x}, \underline{0})=\widetilde{J}_{p / 2-1}(|\underline{x}|)+\underline{x} \widetilde{J}_{p / 2}(|\underline{x}|),
$$

we also have that $c_{0}=1$. By means of induction, one can prove that in this case the coefficients $c_{s}$ take the form

$$
c_{2 \ell}=\frac{(-1)^{\ell} \Gamma\left(\frac{q}{2}\right)}{\Gamma\left(\frac{q}{2}+\ell\right) 2^{2 \ell} \ell !} \quad \text { and } \quad c_{2 \ell+1}=\frac{(-1)^{\ell} \Gamma\left(\frac{q}{2}\right)}{\Gamma\left(\frac{q}{2}+\ell+1\right) 2^{2 \ell+1} \ell !} .
$$

The question is to determine $f(\underline{0}, \underline{y})$. Hereto we start with the following result.

Theorem 3.2. One has

$$
\partial_{\underline{x}}^{s}\left[\widetilde{J}_{p / 2-1}(|\underline{x}|)+\underline{x} \widetilde{J}_{p / 2}(|\underline{x}|)\right]=(-1)^{s}\left(\widetilde{J}_{p / 2-1}(|\underline{x}|)+\underline{x} \widetilde{J}_{p / 2}(|\underline{x}|)\right) .
$$

Proof. By means of the series expansion (2.3), we obtain for the Bessel function

$$
\begin{aligned}
\widetilde{J}_{p / 2-1}(|\underline{x}|) & +\underline{x} \widetilde{J}_{p / 2}(|\underline{x}|) \\
= & 2^{1-p / 2} \sum_{\ell=0}^{\infty} \frac{\underline{x}^{2 \ell}}{\ell ! \Gamma\left(\frac{p}{2}+\ell\right) 2^{2 \ell}}+2^{-p / 2} \sum_{\ell=0}^{\infty} \frac{\underline{x}^{2 \ell+1}}{\ell ! \Gamma\left(\frac{p}{2}+\ell+1\right) 2^{2 \ell}} .
\end{aligned}
$$

Using the fundamental formula $(2.2)$ and substituting $\ell^{\prime}=\ell-1$ into the first series, we find

$$
\begin{aligned}
& \partial_{\underline{x}}\left[\widetilde{J}_{p / 2-1}(|\underline{x}|)+\underline{x}_{p / 2}(|\underline{x}|)\right] \\
& \quad=-2^{1-p / 2} \sum_{\ell=1}^{\infty} \frac{\underline{x}^{2 \ell-1}}{(\ell-1) ! \Gamma\left(\frac{p}{2}+\ell\right) 2^{2 \ell-1}}-2^{-p / 2+1} \sum_{\ell=0}^{\infty} \frac{\underline{x}^{2 \ell}}{\ell ! \Gamma\left(\frac{p}{2}+\ell\right) 2^{2 \ell}} \\
& \quad=-2^{1-p / 2} \sum_{\ell^{\prime}=0}^{\infty} \frac{\underline{x}^{2 \ell^{\prime}+1}}{\ell^{\prime} ! \Gamma\left(\frac{p}{2}+\ell^{\prime}+1\right) 2^{2 \ell^{\prime}+1}}-2^{-p / 2+1} \sum_{\ell=0}^{\infty} \frac{\underline{x}^{2 \ell}}{\ell ! \Gamma\left(\frac{p}{2}+\ell\right) 2^{2 \ell}} \\
& \quad=-\left(\widetilde{J}_{p / 2-1}(|\underline{x}|)+\underline{x}_{p / 2}(|\underline{x}|)\right)
\end{aligned}
$$

which yields the desired result.

Hence, we have

$$
f(\underline{x}, \underline{y})=\sum_{s=0}^{\infty} c_{s} \underline{y}^{s}(-1)^{s}\left(\widetilde{J}_{p / 2-1}(|\underline{x}|)+\underline{x} \widetilde{J}_{p / 2}(|\underline{x}|)\right)
$$


and thus

$$
\begin{aligned}
f(\underline{0}, \underline{y}) & =\sum_{s=0}^{\infty} c_{s} \underline{y}^{s}(-1)^{s} \frac{2^{1-p / 2}}{\Gamma\left(\frac{p}{2}\right)} \\
& =\frac{2^{1-p / 2}}{\Gamma\left(\frac{p}{2}\right)}\left(\sum_{\ell=0}^{\infty} c_{2 \ell} \underline{y}^{2 \ell}-\sum_{\ell=0}^{\infty} c_{2 \ell+1} \underline{y}^{2 \ell+1}\right) \\
& =2^{1-p / 2} \frac{\Gamma\left(\frac{q}{2}\right)}{\Gamma\left(\frac{p}{2}\right)}\left(\sum_{\ell=0}^{\infty} \frac{|\underline{y}|^{2 \ell}}{\Gamma\left(\frac{q}{2}+\ell\right) 2^{2 \ell} \ell !}-\underline{y} \sum_{\ell=0}^{\infty} \frac{|\underline{y}|^{2 \ell}}{\Gamma\left(\frac{q}{2}+\ell+1\right) 2^{2 \ell+1} \ell !}\right) .
\end{aligned}
$$

We now obtain different expressions for $f(\underline{0}, \underline{y})$.

Theorem 3.3. One has that

$$
\begin{aligned}
f(\underline{0}, \underline{y}) & =2^{(q-p) / 2} \frac{\Gamma\left(\frac{q}{2}\right)}{\Gamma\left(\frac{p}{2}\right)}\left(\widetilde{J}_{q / 2-1}(i|\underline{y}|)-\widetilde{J}_{q / 2}(i|\underline{y}|) \underline{y}\right) \\
& =2^{(q-p) / 2} \frac{\Gamma\left(\frac{q}{2}\right)}{\Gamma\left(\frac{p}{2}\right)}|\underline{y}|^{1-q / 2}\left(I_{q / 2-1}(|\underline{y}|)-\frac{\underline{y}}{|\underline{y}|} I_{q / 2}(|\underline{y}|)\right) \\
& =\frac{2^{1-p / 2}}{\Gamma\left(\frac{p}{2}\right)}\left({ }_{0} F_{1}\left(\frac{q}{2} ; \frac{|\underline{y}|^{2}}{4}\right)-\frac{y}{q}{ }_{0} F_{1}\left(\frac{q}{2}+1 ; \frac{|\underline{y}|^{2}}{4}\right)\right)
\end{aligned}
$$

with $I_{\nu}(z)$ being the modified Bessel function of the first kind and ${ }_{0} F_{1}(\beta ; z)$ being the hypergeometric function.

Proof. This theorem is proved using the series expansion (2.3) and consecutively the following relations (see e.g., [10])

$$
\widetilde{J}_{\nu}(z)=z^{-\nu} J_{\nu}(z), \quad J_{\nu}(i z)=i^{\nu} I_{\nu}(z), \quad{ }_{0} F_{1}(\beta ; z)=\sum_{\ell=0}^{\infty} \frac{\Gamma(\beta)}{\Gamma(\beta+\ell)} \frac{z^{\ell}}{\ell !} .
$$

Remark 3.4. During the calculations we have also obtained a closed form for the full biaxial CK-extension $f(\underline{x}, \underline{y})$ :

$$
\begin{gathered}
f(\underline{x}, \underline{y})=2^{q / 2-1} \Gamma\left(\frac{q}{2}\right)\left(\widetilde{J}_{q / 2-1}(i|\underline{y}|)-\widetilde{J}_{q / 2}(i|\underline{y}|) \underline{y}\right)\left(\widetilde{J}_{p / 2-1}(|\underline{x}|)+\underline{x} \widetilde{J}_{p / 2}(|\underline{x}|)\right) \\
=2^{q / 2-1} \Gamma\left(\frac{q}{2}\right)|\underline{y}|^{1-q / 2}\left(I_{q / 2-1}(|\underline{y}|)-\frac{\underline{y}}{|\underline{y}|} I_{q / 2}(|\underline{y}|)\right) \\
\times|\underline{x}|^{1-p / 2}\left(J_{p / 2-1}(|\underline{x}|)+\frac{\underline{x}}{|\underline{x}|} J_{p / 2}(|\underline{x}|)\right) .
\end{gathered}
$$

Note that from the monogenicity of $f$ it follows that the vector part of $f$

$$
[f(\underline{x}, \underline{y})]_{1}=2^{q / 2-1} \Gamma\left(\frac{q}{2}\right)\left(\widetilde{J}_{p / 2}(|\underline{x}|) \widetilde{J}_{q / 2-1}(i|\underline{y}|) \underline{x}-\widetilde{J}_{p / 2-1}(|\underline{x}|) \widetilde{J}_{q / 2}(i|\underline{y}|) \underline{y}\right)
$$

and the parabivector part of $f$

$$
\begin{aligned}
{[f(\underline{x}, \underline{y})]_{0} } & +[f(\underline{x}, \underline{y})]_{2} \\
= & 2^{q / 2-1} \Gamma\left(\frac{q}{2}\right)\left(\widetilde{J}_{p / 2-1}(|\underline{x}|) \widetilde{J}_{q / 2-1}(i|\underline{y}|)+\widetilde{J}_{p / 2}(|\underline{x}|) \widetilde{J}_{q / 2}(i|\underline{y}|) \underline{x} \underline{y}\right)
\end{aligned}
$$


are also monogenic.

\subsection{Third example: Kummer's function}

We now determine the biaxial CK-extension of Kummer's function

$$
{ }_{1} F_{1}\left(a ; c ;-\frac{|\underline{x}|^{2}}{2}\right)=\frac{\Gamma(c)}{\Gamma(a)} \sum_{n=0}^{\infty} \frac{\Gamma(a+n)}{\Gamma(c+n)} \frac{1}{n !}\left(-\frac{|\underline{x}|^{2}}{2}\right)^{n}
$$

with $a, c \in \mathbb{R}$. Like before, we put

$$
f(\underline{x}, \underline{y})=\sum_{s=0}^{\infty} c_{s} \underline{y}^{s} \partial_{\underline{x}}^{s}\left[{ }_{1} F_{1}\left(a ; c ;-\frac{|\underline{x}|^{2}}{2}\right)\right],
$$

where the coefficients $c_{s}$ take the form (3.2).

We start with the following result.

Proposition 3.5. For $n \in \mathbb{N}$ one has

$$
\begin{aligned}
& \partial_{\underline{x}}^{2 n}\left[{ }_{1} F_{1}\left(a ; c ;-\frac{|\underline{x}|^{2}}{2}\right)\right]=\sum_{i=0}^{n} \frac{(a)_{2 n-i}}{(c)_{2 n-i}} 2^{i}\left(\frac{p}{2}+n-i\right)_{i}\left(\begin{array}{c}
n \\
i
\end{array}\right) \\
& \times{ }_{1} F_{1}\left(a+2 n-i ; c+2 n-i ;-\frac{|\underline{x}|^{2}}{2}\right)(-\underline{x})^{2 n-2 i}, \\
& \partial_{\underline{x}}^{2 n+1}\left[{ }_{1} F_{1}\left(a ; c ;-\frac{|\underline{x}|^{2}}{2}\right)\right]=\sum_{i=0}^{n} \frac{(a)_{2 n+1-i}}{(c)_{2 n+1-i}} 2^{i}\left(\frac{p}{2}+n-i+1\right)_{i}\left(\begin{array}{c}
n \\
i
\end{array}\right) \\
& \times{ }_{1} F_{1}\left(a+2 n+1-i ; c+2 n+1-i ;-\frac{|\underline{x}|^{2}}{2}\right)(-\underline{x})^{2 n+1-2 i} .
\end{aligned}
$$

Proof. This result is proved by induction on $n$ using the relations

$$
\partial_{\underline{x}}\left[{ }_{1} F_{1}\left(\alpha ; \beta ;-\frac{|\underline{x}|^{2}}{2}\right)\right]=\frac{\alpha}{\beta}{ }_{1} F_{1}\left(\alpha+1 ; \beta+1 ;-\frac{|\underline{x}|^{2}}{2}\right)(-\underline{x})
$$

and

$$
\partial_{\underline{x}}\left[(-\underline{x})^{\ell}\right]= \begin{cases}\ell(-\underline{x})^{\ell-1} & \text { for } \ell \text { even, } \\ (\ell+p-1)(-\underline{x})^{\ell-1} & \text { for } \ell \text { odd. }\end{cases}
$$

Taking the restriction to $\underline{x}=\underline{0}$ of the differential equations in Proposition 3.5 yields

$$
\begin{aligned}
\partial_{\underline{x}}^{2 n}\left[{ }_{1} F_{1}\left(a ; c ;-\frac{|\underline{x}|^{2}}{2}\right)\right]_{\left.\right|_{\underline{x}=\underline{0}}} & =\frac{(a)_{n}}{(c)_{n}} 2^{n}\left(\frac{p}{2}\right)_{n}, \\
\partial_{\underline{x}}^{2 n+1}\left[{ }_{1} F_{1}\left(a ; c ;-\frac{|\underline{x}|^{2}}{2}\right)\right]_{\left.\right|_{\underline{x}=\underline{0}}} & =0 .
\end{aligned}
$$


Hence, we find

$$
\begin{aligned}
f(\underline{0}, \underline{y}) & =\sum_{n=0}^{\infty} c_{2 n} \underline{y}^{2 n} \frac{(a)_{n}}{(c)_{n}} 2^{n}\left(\frac{p}{2}\right)_{n}=\sum_{n=0}^{\infty} \frac{(a)_{n}\left(\frac{p}{2}\right)_{n}}{(c)_{n}\left(\frac{q}{2}\right)_{n}} \frac{1}{n !}\left(\frac{|\underline{y}|^{2}}{2}\right)^{n} \\
& ={ }_{2} F_{2}\left(a, \frac{p}{2} ; c, \frac{q}{2} ; \frac{|\underline{y}|^{2}}{2}\right) .
\end{aligned}
$$

Remark 3.6. The restriction $f(\underline{0}, \underline{y})$ is again a Kummer function, i.e., we obtain a closed class of functions, in the following four cases

1. $p=q$ :

$$
f(\underline{0}, \underline{y})={ }_{1} F_{1}\left(a ; c ; \frac{|\underline{y}|^{2}}{2}\right)
$$

2. $c=\frac{p}{2}:$

$$
f(\underline{0}, \underline{y})={ }_{1} F_{1}\left(a ; \frac{q}{2} ; \frac{|\underline{y}|^{2}}{2}\right)
$$

3. $a=\frac{q}{2}$ :

$$
f(\underline{0}, \underline{y})={ }_{1} F_{1}\left(\frac{p}{2} ; c ; \frac{|y|^{2}}{2}\right)
$$

4. $a=c$ :

$$
f(\underline{0}, \underline{y})={ }_{1} F_{1}\left(\frac{p}{2} ; \frac{q}{2} ; \frac{|y|^{2}}{2}\right)
$$

Note that in the latter case $f(\underline{x}, \underline{0})=e^{-|\underline{x}|^{2} / 2}$, hence this is the example we have considered in subsection 3.1.

Remark 3.7. The above calculations can be generalized to:

$$
f(\underline{x}, \underline{0})={ }_{k} F_{\ell}\left(a_{1}, \ldots, a_{k} ; c_{1}, \ldots, c_{\ell} ;-\frac{|\underline{x}|^{2}}{2}\right)
$$

with $a_{i} \in \mathbb{R}(i=1, \ldots, k), c_{j} \in \mathbb{R}(j=1, \ldots, \ell)$. In a similar way as above, we obtain

$$
f(\underline{0}, \underline{y})={ }_{k+1} F_{\ell+1}\left(\frac{p}{2}, a_{1}, \ldots, a_{k} ; \frac{q}{2}, c_{1}, \ldots, c_{\ell} ; \frac{|\underline{y}|^{2}}{2}\right) .
$$

\section{Monogenic plane waves}

\subsection{General method}

For this method we refer to [15]. We first consider the axial case.

Let $h(z)$ be a holomorphic function of $z \in \mathbb{C}$. Consider

$$
(1-i \underline{t}) h\left(x_{0}+i\langle\underline{x}, \underline{t}\rangle\right)
$$

with $x_{0} \in \mathbb{R}, \underline{x} \in \mathbb{R}^{m}, \underline{t} \in S^{m-1}$. We have

$$
\left(\partial_{x_{0}}+\partial_{\underline{x}}\right)\left[(1-i \underline{t}) h\left(x_{0}+i\langle\underline{x}, \underline{t}\rangle\right)\right]=h^{\prime}\left(x_{0}+i\langle\underline{x}, \underline{t}\rangle\right)(1+i \underline{t})(1-i \underline{t})=0 .
$$


Hence, $(1-i \underline{t}) h\left(x_{0}+i\langle\underline{x}, \underline{t}\rangle\right)$ is monogenic in $\left(x_{0}, \underline{x}\right)$ and it is a function of $\langle\underline{x}, \underline{t}\rangle$, i.e., it is a monogenic plane wave.

From the above, it is clear that the axial CK-extension of $h\left(x_{0}\right)$ can be written in the following integral form:

$$
\mathrm{CK}(h)\left(x_{0}, \underline{x}\right)=\frac{\Gamma\left(\frac{m}{2}\right)}{2 \pi^{m / 2}} \int_{S^{m-1}}(1-i \underline{t}) h\left(x_{0}+i\langle\underline{x}, \underline{t}\rangle\right) d \underline{t} .
$$

For the functions

$$
h\left(x_{0}\right)=e^{x_{0}}, \quad h\left(x_{0}\right)=e^{-x_{0}^{2} / 2}, \quad \text { and } \quad h\left(x_{0}\right)=\left(1-x_{0}^{2}\right)^{\alpha}, \alpha \in \mathbb{R},
$$

we know the restriction $\operatorname{CK}(h)(0, \underline{x})$ from subsection 2.2 .

On the other hand, we have

$$
\mathrm{CK}(h)(0, \underline{x})=\frac{\Gamma\left(\frac{m}{2}\right)}{2 \pi^{m / 2}} \int_{S^{m-1}}(1-i \underline{t}) h(i\langle\underline{x}, \underline{t}\rangle) d \underline{t} .
$$

By applying on the right hand side of the above equation the following FunkHecke theorem (see for e.g., [7]),

Theorem 4.1 (Funk-Hecke Theorem). Let $S_{k}$ be a spherical harmonic of degree $k$, then

$$
\int_{S^{m-1}} f(\langle\underline{\omega}, \underline{\eta}\rangle) S_{k}(\underline{\omega}) d S(\underline{\omega})=A_{m-1} S_{k}(\underline{\eta}) \int_{-1}^{1} f(t)\left(1-t^{2}\right)^{(m-3) / 2} P_{k, m}(t) d t
$$

where $P_{k, m}(t)$ denotes the Legendre polynomial of degree $k$ in $\mathbb{R}^{m}$, expressed as follows in terms of the Gegenbauer polynomials

$$
P_{k, m}(t)=\frac{k !(m-3) !}{(k+m-3) !} C_{k}^{(m-2) / 2}(t),
$$

and $A_{m-1}=\frac{2 \pi^{(m-1) / 2}}{\Gamma\left(\frac{m-1}{2}\right)}$ the area of the unit sphere $S^{m-2}$ in $\mathbb{R}^{m-1}$.

we will be able to obtain integral expressions for $\operatorname{CK}(h)(0, \underline{x})$. In other words, the aim is to derive integral representations for special functions of classical analysis by using Clifford analysis techniques.

Remark 4.2. The above idea can also be applied to obtain an integral expression for the full axial CK-extension $\operatorname{CK}(h)\left(x_{0}, \underline{x}\right)$.

In the biaxial case monogenic plane waves are of the form

$$
h(i\langle\underline{x}, \underline{t}\rangle-\langle\underline{y}, \underline{s}\rangle)(\underline{t}+i \underline{s}), \quad \underline{t} \in S^{p-1}, \underline{s} \in S^{q-1}
$$

giving vector-valued monogenics, and of the form

$$
h(i\langle\underline{x}, \underline{t}\rangle-\langle\underline{y}, \underline{s}\rangle)(1+i \underline{t s}), \quad \underline{t} \in S^{p-1}, \underline{s} \in S^{q-1}
$$

giving parabivector-valued monogenics. The integration takes place over the product of spheres $S^{p-1} \times S^{q-1}$ and to evaluate the integrals one has to apply the Funk-Hecke theorem twice.

Let us consider the restrictions for the parabivector-valued monogenics:

$$
f^{p}(\underline{x}, \underline{y})=\int_{S^{p-1}} \int_{S^{q-1}} h(i\langle\underline{x}, \underline{t}\rangle-\langle\underline{y}, \underline{s}\rangle)(1+i \underline{t} \underline{s}) d \underline{t} d \underline{s} .
$$


First, we have that

$$
\begin{aligned}
f^{p}(\underline{0}, \underline{y}) & =\frac{2 \pi^{p / 2}}{\Gamma\left(\frac{p}{2}\right)} \int_{S^{q-1}} h(-\langle\underline{y}, \underline{s}\rangle) d \underline{s} \\
& =\frac{4 \pi^{(p+q-1) / 2}}{\Gamma\left(\frac{p}{2}\right) \Gamma\left(\frac{q-1}{2}\right)} \int_{-1}^{1} h(-|\underline{y}| t)\left(1-t^{2}\right)^{(q-3) / 2} d t,
\end{aligned}
$$

while the restriction to $\underline{y}=\underline{0}$ takes the form

$$
\begin{aligned}
f^{p}(\underline{x}, \underline{0}) & =\frac{2 \pi^{q / 2}}{\Gamma\left(\frac{q}{2}\right)} \int_{S^{p-1}} h(i\langle\underline{x}, \underline{t}\rangle) d \underline{t} \\
& =\frac{4 \pi^{(p+q-1) / 2}}{\Gamma\left(\frac{p-1}{2}\right) \Gamma\left(\frac{q}{2}\right)} \int_{-1}^{1} h(i|\underline{x}| t)\left(1-t^{2}\right)^{(p-3) / 2} d t .
\end{aligned}
$$

In a similar way, we obtain for the vector-valued monogenics

$$
f^{v}(\underline{x}, \underline{y})=\int_{S^{p-1}} \int_{S^{q-1}} h(i\langle\underline{x}, \underline{t}\rangle-\langle\underline{y}, \underline{s}\rangle)(\underline{t}+i \underline{s}) d \underline{t} d \underline{s}
$$

the following results:

$$
\begin{aligned}
& f^{v}(\underline{0}, \underline{y})=\frac{i 4 \pi^{(p+q-1) / 2}}{\Gamma\left(\frac{p}{2}\right) \Gamma\left(\frac{q-1}{2}\right)} \frac{\underline{y}}{|\underline{y}|} \int_{-1}^{1} h(-|\underline{y}| t)\left(1-t^{2}\right)^{(q-3) / 2} t d t, \\
& f^{v}(\underline{x}, \underline{0})=\frac{4 \pi^{(p+q-1) / 2}}{\Gamma\left(\frac{q}{2}\right) \Gamma\left(\frac{p-1}{2}\right)} \frac{\underline{x}}{|\underline{x}|} \int_{-1}^{1} h(i|\underline{x}| t)\left(1-t^{2}\right)^{(p-3) / 2} t d t .
\end{aligned}
$$

4.2. The axial case: method applied to $\operatorname{CK}(h)(0, \underline{x})$

4.2.1. $h\left(x_{0}\right)=e^{-x_{0}^{2} / 2}$. In this case, we know from subsection 2.2 .2 that

$$
\mathrm{CK}(h)(0, \underline{x})={ }_{1} F_{1}\left(\frac{1}{2} ; \frac{m}{2} ; \frac{|\underline{x}|^{2}}{2}\right) .
$$

On the other hand, using spherical coordinates $\underline{x}=r \underline{\omega}, r=|\underline{x}|, \underline{\omega} \in S^{m-1}$ and the Funk-Hecke theorem, we have that

$$
\begin{aligned}
\mathrm{CK}(h)(0, \underline{x}) & =\frac{\Gamma\left(\frac{m}{2}\right)}{2 \pi^{m / 2}} \int_{S^{m-1}}(1-i \underline{t}) e^{\langle\underline{x}, \underline{t}\rangle^{2} / 2} d \underline{t} \\
& =\frac{\Gamma\left(\frac{m}{2}\right)}{\sqrt{\pi} \Gamma\left(\frac{m-1}{2}\right)}\left(\int_{-1}^{1} e^{r^{2} t^{2} / 2}\left(1-t^{2}\right)^{(m-3) / 2} d t\right. \\
& \left.-i \underline{\omega} \int_{-1}^{1} e^{r^{2} t^{2} / 2}\left(1-t^{2}\right)^{(m-3) / 2} t d t\right) \\
& =\frac{2 \Gamma\left(\frac{m}{2}\right)}{\sqrt{\pi} \Gamma\left(\frac{m-1}{2}\right)} \int_{0}^{1} e^{|\underline{x}|^{2} t^{2} / 2}\left(1-t^{2}\right)^{(m-3) / 2} d t \\
& =\frac{\Gamma\left(\frac{m}{2}\right)}{\sqrt{\pi} \Gamma\left(\frac{m-1}{2}\right)} \int_{0}^{1} e^{|\underline{x}|^{2} t / 2}(1-t)^{(m-3) / 2} t^{-\frac{1}{2}} d t
\end{aligned}
$$


where in the last step we have substituted $t^{\prime}=t^{2}$.

Hence, we have obtained the following integral representation for Kummer's function

$$
{ }_{1} F_{1}\left(\frac{1}{2} ; \frac{m}{2} ; z\right)=\frac{\Gamma\left(\frac{m}{2}\right)}{\sqrt{\pi} \Gamma\left(\frac{m-1}{2}\right)} \int_{0}^{1} e^{z t}(1-t)^{(m-3) / 2} t^{-\frac{1}{2}} d t .
$$

This integral expression can also be found, for example, in [10, p. 274].

4.2.2. $h\left(x_{0}\right)=e^{x_{0}}$. On the one hand, we know from subsection 2.2 .1 that

$$
\operatorname{CK}(h)(0, \underline{x})=\Gamma\left(\frac{m}{2}\right) 2^{m / 2-1}\left(\widetilde{J}_{m / 2-1}(|\underline{x}|)+\widetilde{J}_{m / 2}(|\underline{x}|) \underline{x}\right),
$$

while on the other hand, using the monogenic plane wave approach we obtain

$$
\begin{aligned}
\operatorname{CK}(h)(0, \underline{x})= & \frac{\Gamma\left(\frac{m}{2}\right)}{2 \pi^{m / 2}}\left(\int_{S^{m-1}} e^{i|\underline{x}|\langle\underline{\omega}, \underline{\underline{t}}\rangle} d \underline{t}-i \int_{S^{m-1}} \underline{t} e^{i|\underline{x}|\langle\underline{\omega}, \underline{t}\rangle} d \underline{t}\right) \\
= & \frac{\Gamma\left(\frac{m}{2}\right)}{\sqrt{\pi} \Gamma\left(\frac{m-1}{2}\right)}\left(\int_{-1}^{1} e^{i|\underline{x}| t}\left(1-t^{2}\right)^{(m-3) / 2} d t\right. \\
& \left.\quad-i \underline{\omega} \int_{-1}^{1} e^{i|\underline{x}| t}\left(1-t^{2}\right)^{(m-3) / 2} t d t\right) .
\end{aligned}
$$

Equalizing the scalar and vector part of (4.1) and (4.2) yields

$$
|\underline{x}|^{1-m / 2} J_{m / 2-1}(|\underline{x}|)=\frac{2^{1-m / 2}}{\sqrt{\pi} \Gamma\left(\frac{m-1}{2}\right)} \int_{-1}^{1} e^{i|\underline{x}| t}\left(1-t^{2}\right)^{(m-3) / 2} d t
$$

and

$$
|\underline{x}|^{1-m / 2} J_{m / 2}(|\underline{x}|)=\frac{2^{1-m / 2}(-i)}{\sqrt{\pi} \Gamma\left(\frac{m-1}{2}\right)} \int_{-1}^{1} e^{i|\underline{x}| t}\left(1-t^{2}\right)^{(m-3) / 2} t d t .
$$

Expression (4.3) is an application of Poisson's integral (see e.g. [4, p. 81]):

$$
z^{-\nu} J_{\nu}(z)=\frac{2^{-\nu}}{\sqrt{\pi} \Gamma\left(\nu+\frac{1}{2}\right)} \int_{-1}^{1} e^{i z t}\left(1-t^{2}\right)^{\nu-\frac{1}{2}} d t, \quad \operatorname{Re}(\nu)>-\frac{1}{2}
$$

and formula (4.4) is an application of the following integral expression which is derived in [7, pp. 203-204]:

$$
\begin{aligned}
& J_{n+m / 2-1}(z)= \\
& \frac{(-i)^{n}}{\sqrt{\pi} \Gamma\left(\frac{m-1}{2}\right)}\left(\frac{z}{2}\right)^{m / 2-1} \int_{-1}^{1} e^{i z t} P_{n, m}(t)\left(1-t^{2}\right)^{(m-3) / 2} d t .
\end{aligned}
$$


4.2.3. $h\left(x_{0}\right)=\left(1-x_{0}^{2}\right)^{\alpha}, \alpha \in \mathbb{R}$. Let us now consider the last function for which we know $\operatorname{CK}(h)(0, \underline{x})$ from subsection 2.2 , namely the Gegenbauer weight function $h\left(x_{0}\right)=\left(1-x_{0}^{2}\right)^{\alpha}, \alpha \in \mathbb{R}$.

Applying the monogenic plane wave approach we find that

$$
\begin{aligned}
{ }_{2} F_{1}\left(-\alpha, \frac{1}{2} ; \frac{m}{2} ;-|\underline{x}|^{2}\right)= & \frac{\Gamma\left(\frac{m}{2}\right)}{2 \pi^{m / 2}}\left(\int_{S^{m-1}}\left(1+|\underline{x}|^{2}\langle\underline{\omega}, \underline{t}\rangle^{2}\right)^{\alpha} d \underline{t}\right. \\
& \left.\quad-i \int_{S^{m-1}} \underline{t}\left(1+|\underline{x}|^{2}\langle\underline{\omega}, \underline{t}\rangle^{2}\right)^{\alpha} d \underline{t}\right) \\
= & \frac{\Gamma\left(\frac{m}{2}\right)}{\sqrt{\pi} \Gamma\left(\frac{m-1}{2}\right)}\left(\int_{-1}^{1}\left(1+|\underline{x}|^{2} t^{2}\right)^{\alpha}\left(1-t^{2}\right)^{(m-3) / 2} d t\right. \\
= & \frac{2 \Gamma\left(\frac{m}{2}\right)}{\sqrt{\pi} \Gamma\left(\frac{m-1}{2}\right)} \int_{0}^{1}\left(1+|\underline{x}|^{2} t^{2}\right)^{\alpha}\left(1-t^{2}\right)^{(m-3) / 2} d t \\
= & \left.\frac{\Gamma\left(\frac{m}{2}\right)}{\sqrt{\pi} \Gamma\left(\frac{m-1}{2}\right)} \int_{0}^{1}\left(1+|\underline{x}|^{2} t^{2}\right)^{\alpha}\left(1-t^{2}\right)^{(m-3) / 2} t d t\right)
\end{aligned}
$$

where in the last line we have substituted $t^{\prime}=t^{2}$. This is a special case of the following integral representation of hypergeometric functions, which can for e.g. be found in [10, p. 54]:

$$
{ }_{2} F_{1}(a, b ; c ; z)=\frac{\Gamma(c)}{\Gamma(b) \Gamma(c-b)} \int_{0}^{1} t^{b-1}(1-t)^{c-b-1}(1-t z)^{-a} d t
$$

$\operatorname{Re}(c)>\operatorname{Re}(b)>0,|\arg (1-z)|<\pi$.

\subsection{The axial case: method applied to the full axial CK-extension}

4.3.1. $h\left(x_{0}\right)=e^{x_{0}}$. It is easily seen that this leads to the same integral equations as in subsection 4.2.2. 
4.3.2. $h\left(x_{0}\right)=e^{-x_{0}^{2} / 2}$. For the Hermite weight function the full axial CKextension takes the form

$$
\begin{aligned}
& f\left(x_{0}, \underline{x}\right)=\frac{\Gamma\left(\frac{m}{2}\right)}{2 \pi^{m / 2}} e^{-\frac{1}{2} x_{0}^{2}}\left(\int_{S^{m-1}} e^{\frac{1}{2}|\underline{x}|^{2}\langle\underline{\omega}, \underline{t}\rangle^{2}} e^{-i x_{0}|\underline{x}|\langle\underline{\omega}, \underline{t}\rangle} d \underline{t}\right. \\
& \left.-i \int_{S^{m-1}} \underline{t} e^{\frac{1}{2}|\underline{x}|^{2}\langle\underline{\omega}, \underline{t}\rangle^{2}} e^{-i x_{0}|\underline{x}|\langle\underline{\omega}, \underline{t}\rangle} d \underline{t}\right) \\
& =\frac{\Gamma\left(\frac{m}{2}\right)}{\sqrt{\pi} \Gamma\left(\frac{m-1}{2}\right)} e^{-\frac{1}{2} x_{0}^{2}} \\
& \times\left(\int_{-1}^{1} e^{\frac{1}{2}|\underline{x}|^{2} t^{2}}\left(\cos \left(x_{0}|\underline{x}| t\right)-i \sin \left(x_{0}|\underline{x}| t\right)\right)\left(1-t^{2}\right)^{(m-3) / 2} d t\right. \\
& \left.-i \underline{\omega} \int_{-1}^{1} e^{\frac{1}{2}|\underline{x}|^{2} t^{2}}\left(\cos \left(x_{0}|\underline{x}| t\right)-i \sin \left(x_{0}|\underline{x}| t\right)\right)\left(1-t^{2}\right)^{(m-3) / 2} t d t\right) \\
& =\frac{2 \Gamma\left(\frac{m}{2}\right)}{\sqrt{\pi} \Gamma\left(\frac{m-1}{2}\right)} e^{-\frac{1}{2} x_{0}^{2}}\left(\int_{0}^{1} e^{\frac{1}{2}|\underline{x}|^{2} t^{2}} \cos \left(x_{0}|\underline{x}| t\right)\left(1-t^{2}\right)^{(m-3) / 2} d t\right. \\
& \left.-\underline{\omega} \int_{0}^{1} e^{\frac{1}{2}|\underline{x}|^{2} t^{2}} \sin \left(x_{0}|\underline{x}| t\right)\left(1-t^{2}\right)^{(m-3) / 2} t d t\right) .
\end{aligned}
$$

In order to obtain integral expressions from the comparison of the above expression with (2.7), we expand the cosine- and sine function and moreover execute the substitution $u=t^{2}$, yielding:

$$
\begin{aligned}
& f\left(x_{0}, \underline{x}\right)= \\
& \frac{\Gamma\left(\frac{m}{2}\right)}{\sqrt{\pi} \Gamma\left(\frac{m-1}{2}\right)} e^{-\frac{1}{2} x_{0}^{2}}\left(\sum_{\ell=0}^{\infty} \frac{(-1)^{\ell}}{(2 \ell) !} x_{0}^{2 \ell}|\underline{x}|^{2 \ell} \int_{0}^{1} e^{\frac{1}{2}|\underline{x}|^{2} u} u^{\ell-\frac{1}{2}}(1-u)^{(m-3) / 2} d u\right. \\
& \left.\quad-\underline{\omega} \sum_{\ell=0}^{\infty} \frac{(-1)^{\ell}}{(2 \ell+1) !} x_{0}^{2 \ell+1}|\underline{x}|^{2 \ell+1} \int_{0}^{1} e^{\frac{1}{2}|\underline{x}|^{2} u} u^{\ell+\frac{1}{2}}(1-u)^{(m-3) / 2} d u\right) .
\end{aligned}
$$

By comparing the above expression with (2.7), we obtain the following two integral equations:

$$
{ }_{1} F_{1}\left(\ell+\frac{1}{2} ; \frac{m}{2}+\ell ; \frac{|\underline{x}|^{2}}{2}\right)=\frac{1}{B\left(\ell+\frac{1}{2}, \frac{m-1}{2}\right)} \int_{0}^{1} e^{\frac{1}{2}|\underline{x}|^{2} u} u^{\ell-\frac{1}{2}}(1-u)^{(m-3) / 2} d u
$$

and

$$
\begin{aligned}
{ }_{1} F_{1}\left(\ell+\frac{3}{2} ; \frac{m}{2}+\ell\right. & \left.+1 ; \frac{|\underline{x}|^{2}}{2}\right) \\
& =\frac{1}{B\left(\ell+\frac{3}{2}, \frac{m-1}{2}\right)} \int_{0}^{1} e^{\frac{1}{2}|\underline{x}|^{2} u} u^{\ell+\frac{1}{2}}(1-u)^{(m-3) / 2} d u,
\end{aligned}
$$


which are applications of (see e.g., [10, p. 274]):

$$
{ }_{1} F_{1}(a ; c ; z)=\frac{1}{B(a, c-a)} \int_{0}^{1} e^{z t} t^{a-1}(1-t)^{c-a-1} d t, \quad \operatorname{Re}(c)>\operatorname{Re}(a)>0 .
$$

\subsection{Monogenic plane wave integrals in the biaxial case}

We will illustrate this method for $h(z)=e^{z}$ in order to obtain integral formulas for the Clifford-Bessel function of biaxial type (see subsection 3.2):

$$
\begin{aligned}
& f^{B e s}(\underline{x}, \underline{y}) \\
& =2^{q / 2-1} \Gamma\left(\frac{q}{2}\right)\left(\widetilde{J}_{q / 2-1}(i|\underline{y}|)-\widetilde{J}_{q / 2}(i|\underline{y}|) \underline{y}\right)\left(\widetilde{J}_{p / 2-1}(|\underline{x}|)+\underline{x} \widetilde{J}_{p / 2}(|\underline{x}|)\right) .
\end{aligned}
$$

Applying Funk-Hecke twice, we obtain for the vector-valued monogenic

$$
\begin{aligned}
& f^{v}(\underline{x}, \underline{y})= \int_{S^{p-1}} \int_{S^{q-1}} e^{i\langle\underline{x}, \underline{t}\rangle-\langle\underline{y}, \underline{s}\rangle}(\underline{t}+i \underline{s}) d \underline{t} d \underline{s} \\
&=\frac{4 \pi^{(p+q) / 2-1}}{\Gamma\left(\frac{p-1}{2}\right) \Gamma\left(\frac{q-1}{2}\right)} \underline{\omega}\left(\int_{-1}^{1} e^{i|\underline{x}| t}\left(1-t^{2}\right)^{(p-3) / 2} t d t\right) \\
& \quad \times\left(\int_{-1}^{1} e^{-|\underline{y}| s}\left(1-s^{2}\right)^{(q-3) / 2} d s\right) \\
&+i \frac{4 \pi^{(p+q) / 2-1}}{\Gamma\left(\frac{p-1}{2}\right) \Gamma\left(\frac{q-1}{2}\right)} \underline{\xi}\left(\int_{-1}^{1} e^{i|\underline{x}| t}\left(1-t^{2}\right)^{(p-3) / 2} d t\right) \\
& \times\left(\int_{-1}^{1} e^{-|\underline{y}| s}\left(1-s^{2}\right)^{(q-3) / 2} s d s\right) .
\end{aligned}
$$

Next, in view of (4.5),

$$
f^{v}(\underline{x}, \underline{y})=i(2 \pi)^{(p+q) / 2}\left(\widetilde{J}_{p / 2}(|\underline{x}|) \widetilde{J}_{q / 2-1}(i|\underline{y}|) \underline{x}-\widetilde{J}_{p / 2-1}(|\underline{x}|) \widetilde{J}_{q / 2}(i|\underline{y}|) \underline{y}\right) .
$$

In a similar way, we find for the parabivector-valued monogenic:

$$
f^{p}(\underline{x}, \underline{y})=(2 \pi)^{(p+q) / 2}\left(\widetilde{J}_{p / 2-1}(|\underline{x}|) \widetilde{J}_{q / 2-1}(i|\underline{y}|)+\underline{x} \underline{y} \widetilde{J}_{p / 2}(|\underline{x}|) \widetilde{J}_{q / 2}(i|\underline{y}|)\right) .
$$

From the above calculations and Remark 3.4, we obtain the following result.

Theorem 4.3. The vector and parabivector part of the Clifford-Bessel function of biaxial type

$$
\begin{aligned}
& f^{\text {Bes }}(\underline{x}, \underline{y}) \\
& \quad=2^{q / 2-1} \Gamma\left(\frac{q}{2}\right)\left(\widetilde{J}_{q / 2-1}(i|\underline{y}|)-\widetilde{J}_{q / 2}(i|\underline{y}|) \underline{y}\right)\left(\widetilde{J}_{p / 2-1}(|\underline{x}|)+\underline{x} \widetilde{J}_{p / 2}(|\underline{x}|)\right)
\end{aligned}
$$

can be written as plane wave integrals:

$$
\left[f^{B e s}(\underline{x}, \underline{y})\right]_{1}=(-i) \frac{\Gamma\left(\frac{q}{2}\right)}{2^{1+p / 2} \pi^{(p+q) / 2}} \int_{S^{p-1}} \int_{S^{q-1}} e^{i\langle\underline{x}, \underline{t}\rangle-\langle\underline{y}, \underline{s}\rangle}(\underline{t}+i \underline{s}) d \underline{t} d \underline{s}
$$


and

$$
\begin{aligned}
{\left[f^{B e s}(\underline{x}, \underline{y})\right]_{0}+\left[f^{B e s}(\underline{x}, \underline{y})\right]_{2} } \\
\quad=\frac{\Gamma\left(\frac{q}{2}\right)}{2^{1+p / 2} \pi^{(p+q) / 2}} \int_{S^{p-1}} \int_{S^{q-1}} e^{i\langle\underline{x}, \underline{t}\rangle-\langle\underline{y}, \underline{s}\rangle}(1+i \underline{t s}) d \underline{t} d \underline{s} .
\end{aligned}
$$

One thus also has that

$$
f^{B e s}(\underline{x}, \underline{y})=\frac{\Gamma\left(\frac{q}{2}\right)}{2^{1+p / 2} \pi^{(p+q) / 2}} \int_{S^{p-1}} \int_{S^{q-1}} e^{i\langle\underline{x}, \underline{t}\rangle-\langle\underline{y}, \underline{s}\rangle}(1-i \underline{t}+\underline{s}+i \underline{t s}) d \underline{t} d \underline{s} .
$$

\section{References}

[1] F. Brackx, R. Delanghe and F. Sommen, Clifford Analysis. Vol. 76 of Research Notes in Mathematics. Pitman, Boston, MA, 1982.

[2] F. Colombo, I. Sabadini and F. Sommen, The Inverse Fueter Mapping Theorem. Communications on Pure and Applied Analysis, 10, No. 4 (2011), 1165-1181.

[3] R. Delanghe, F. Sommen and V. Souček, Clifford Algebra and Spinor-Valued Functions. Kluwer Academic Publishers Group, Dordrecht, 1992.

[4] A. Erdélyi, W. Magnus, F. Oberhettinger and F. G. Tricomi, Higher Transcendental Functions 2. McGraw-Hill, New York, 1953.

[5] J. Gilbert and M. Murray, Clifford Algebras and Dirac Operators in Harmonic Analysis. Cambridge University Press, Cambridge, 1991.

[6] K. Gürlebeck, K. Habetha and W. Sprössig, Holomorphic Functions in the Plane and N-dimensional Space. Birkhäuser, Basel, 2008.

[7] H. Hochstadt, The Functions of Mathematical Physics. In: Pure and Applied Mathematics, Vol. 23, Wiley-Interscience, New York, 1971.

[8] G. Jank and F. Sommen, Clifford Analysis, Biaxial Symmetry and Pseudoanalytic Functions. Complex Var. Elliptic Equ. 13 (3-4) (1990), 195-212.

[9] R. Lávička, A Generalization of Fueter's Monogenic Functions to Fine Domains. Rend. Circ. Mat. di Palermo (2) Suppl. 79 (2006), 129-138.

[10] W. Magnus, F. Oberhettinger and R. P. Soni, Formulas and Theorems for the Special Functions of Mathematical Physics. Springer-Verlag, Berlin, 1966.

[11] D. Peña Peña, T. Qian and F. Sommen, An Alternative Proof of Fueter's Theorem. Complex Var. Elliptic Equ. 51(8-11) (2006), 913-922.

[12] D. Peña Peña and F. Sommen, Monogenic Gaussian Distribution in Closed Form and the Gaussian Fundamental Solution. Complex Var. Elliptic Equ. 54, No. 5 (2009), 429-440.

[13] T. Qian and F. Sommen, Deriving Harmonic Functions in Higher Dimensional Spaces. Z. Anal. Anwendungen 22(2) (2003), 275-288.

[14] F. Sommen, Special Functions in Clifford Analysis and Axial Symmetry. J. Math. Anal. Appl. 130, No. 1 (1988), 110-133. Zbl 0634.30042.

[15] F. Sommen, Clifford Analysis and Integral Geometry. In: Clifford Algebras and their Applications in Mathematical Physics, A. Micali et al., Kluwer, Dordrecht, 1992, 293-311. 
Nele De Schepper

Ghent University - Department of Mathematical Analysis

Galglaan 2

B-9000 Gent, Belgium

e-mail: nds@cage. ugent.be

Frank Sommen

Ghent University - Department of Mathematical Analysis

Galglaan 2

B-9000 Gent, Belgium

e-mail: fs@cage.ugent.be 